\title{
Analysis of Smart Contract using Ethereum Blockchain
}

\author{
Amit Sinha ${ }^{1}$, Harikesh Singh ${ }^{1}$, and Ashwin Perti ${ }^{1}$ \\ ${ }^{1}$ ABES Engineering College
}

November 18, 2020

\begin{abstract}
A blockchain is, in the easiest of terms, a period stepped arrangement of unchanging records of information that is overseen by ia igroup iof iPCs inot ipossessed iby iany isingle ielement. iEvery ione iof ithese isquares iof iinformation iis imade isure iabout iand ibound ito ione ianother iutilizing icryptographic istandards. Ethereum iis ia iworldwide, idecentralized istage ifor icash iand inew isorts iof iutilizations. iOn iEthereum, iyou ican icompose icode ithat icontrols ithe icash, iand imanufacture iapplications iavailable ianyplace ion ithe iplanet.IA Smart iContract iis ia iPC iprogram ithat ilegitimately iand iconsequently icontrols ithe iexchange iof icomputerized iresources ibetween ithe igatherings iunder ispecific iconditions. iA ikeen iagreement iworks isimilarly ias ia iconventional iagreement iwhile ilikewise iconsequently iimplementing ithe iagreement. iSavvy icontracts iare iprograms ithat iexecute iprecisely ias ithey iare iset iup (coded, imodified) iby itheir imakers. iMuch ithe isame ias ia icustomary iagreement iis ienforceable iby ilaw, ishrewd iagreements iare ienforceable iby icode. iA ibrilliant iagreement iis ionly ian iadvanced iagreement iwith ithe isecurity icoding iof ithe iblockchain. iA isavvy icontract ihas isubtleties iand iconsents iwritten iin icode ithat irequire ia iprecise igrouping iof ioccasions ito ihappen ito itrigger ithe iunderstanding iof ithe iterms ireferenced iin ithe ibrilliant iagreement.
\end{abstract}

\section{Hosted file}

pj-2582-6-f1.pdf available at https://authorea.com/users/369088/articles/488018-analysis-ofsmart-contract-using-ethereum-blockchain 